\title{
Cardiac imaging of prosthetic valve dysfunction by transesophageal echocardiography
}

\author{
Melek Zekiye Ulucam
}

Cardiology Department, Baskent University School of Medicine, Ankara, Turkey

Email: melekulucam@gmail.com

Received 15 October 2012; revised 18 November 2012; accepted 12 December 2012

\begin{abstract}
Prosthetic heart valve (PHV) replacement replaces a heart disease with another one. There is no ideal prosthetic heart valve. All of the prosthetic heart valves need to be followed by caution. PHV diseases (PHVD) includes a group of pathologic processes like prosthetic valve obstruction, thromboembolism and regurgitation. Correct diagnosis and treatment of these pathologic situations will decrease mortality and morbidity due to PHVD. Knowledge about PHVD and correct usage of cardiac imaging modalities for the diagnosis, make possible to solve the problem. Most helpful diagnostic test for PHVD is transesophageal echocardiography (TEE). The aim of this review is to describe TEE in PHVD, TEE imaging characteristics of some special PHVD and clinical guidance of TEE in prosthetic heart valve problems.
\end{abstract}

Keywords: Prosthetic Heart Valve; Thrombosis; Thromboemboli; Regurgitation

\section{INTRODUCTION}

Prosthetic heart valves (PHV), used for many years in the treatment of valvular heart diseases. If it is necessary, diseased heart valves can be replaced with mechanical valves, or bioprosthesis, which is generally derived from pig pericardium. Prosthetic valve selection should be made with caution, depending on the patient's clinical characteristics. However, no matter how accurate selection, severe problems may occur during the follow-up of prosthetic valves. While some of these problems inevitably, some of them can be prevented effectively by medical treatment. In any case, in order to be able to get an appropriate approach to the problems of prosthetic valve, we need to know imaging of prosthetic heart valve disease (PHVD) very well. Transesophageal echocardiography (TEE) is the major diagnostic tool which enables to visualise structure of any PHV and most of the pathologies with PHVD. The aim of this review is to describe
TEE findings in PHV diseases, TEE imaging characteristics of special PHVD and clinical guidance of TEE in prosthetic heart valve problems.

\section{PROSTHETIC VALVE OBSTRUCTION}

Prosthetic valve obstruction, may develop due to thrombus, vegetations, pannus formation, or combinations thereof [1]. Most frequent cause [2] of prosthetic mitral valve obstruction is thrombus, while the prosthetic aortic valve is pannus (Figures $\mathbf{1}$ and 2).

The frequency of prosthetic valve obstruction, depends on the valve type and size, location of the valve, adequacy of anticoagulant therapy. It is determined as $0.1 \%$ $0.4 \% /$ year [3]. Decreased exercise capacity, increased shortness of breath, or thromboembolic events in a patient with a mechanical or biological prosthesis, may be the signs of prosthetic valve obstruction. If anticoagulant therapy is insufficient, if the patient is neglected anticoagulant therapy or there is a tendency to clotting, suspicions should increase about prosthetic valve obstruction. To determine the cause of the prosthetic valve obstruction, clinical and laboratory findings should be considered together.

Echocardiography is the most helpful diagnostic test. Transthoracic echocardiography (TTE) is often followed by TEE during the diagnosis period. For diagnosis, maximal and mean pressure gradient and effective valve area for the prosthesis should be measured/calculated and compared with the predicted normal values and the values of the first TTE/TEE of the patient after surgery. It should be noted that, increased flow rate determined on any prosthetic valve may not always indicate prosthetic valve obstruction, it should be evaluated together with other data about the valve [4]. Recent guidelines indicated that, in patients with suspected thrombosis, TTE with Doppler echocardiography should be used to determine the hemodynamic severity, while TEE and fluoroscopy is necessary to see the valve motion and clot properties (Class 1 requirements, level of evidence B) [1]. TEE may be helpful for the differentiation of thrombus and pannus. 


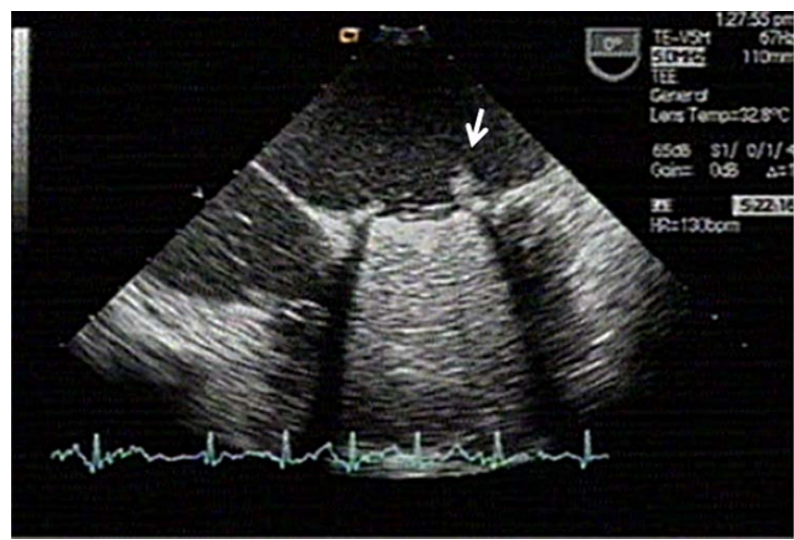

Figure 1. A large left atrial thrombus built-in mechanical prosthesis at mitral position.

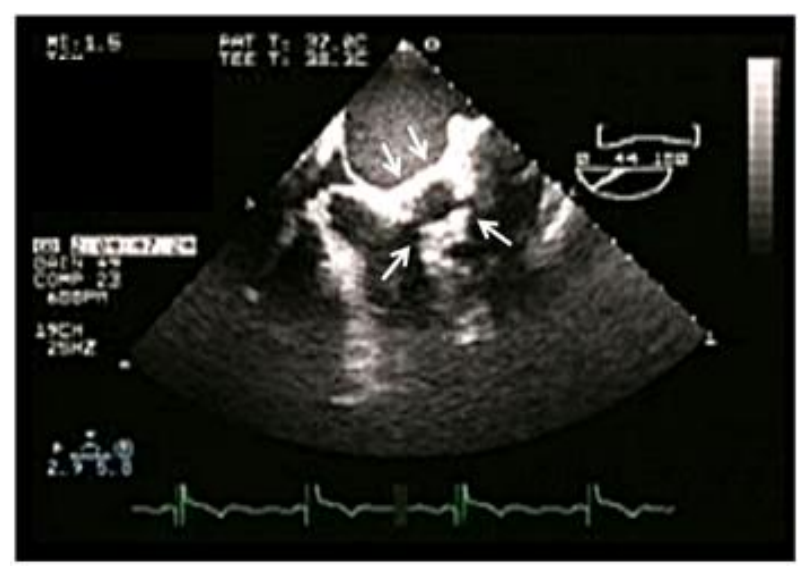

Figure 2. Pannus view at a mechanical prosthetic valve at aortic position (arrowed).

In TEE examination, findings suggestive of thrombus are, a mass with soft echo-density and videointensity ratio $<0.7$, together with the occurrence of inadequate anticoagulant therapy [5].

Prosthetic valve obstruction is treated with medical or surgical methods. All types of treatment involves high risk. Emergency surgery has highest risk ratios. In fibrinolytic therapy, bleeding, systemic embolism, and reobstruction may occur and complicate the treatment period. Differential diagnosis is necessary for decision of best treatment method. In mitral prosthesis, in the presence of pannus, which can be difficult to distinguish from thrombus, or in cases with chronic obstruction, fibrinolytic therapy is unlikely to be successful $[6,7]$. TEE is helpful for differential diagnosis of pannus and thrombus.

\section{PROSTHESIS THROMBOEMBOLISM}

Thromboembolism of prosthetic valves is a common complication [8]. The risk of thromboembolism in tilting single and double disc prosthesis is similar to bioprosthetic valves and it is $1.5 \%-2 \%$ patient/year [8].
The etiology of the thromboembolism after surgery is multifactorial [9]. Several thromboembolic events caused by vegetation, thrombus and abnormal flow conditions of the prosthesis. Chronic atrial fibrillation and PHV dysfunction increase the risk of thromboembolism. In patients with mitral valve prosthesis, if left atrium is small, patient is in sinus rhythm and cardiac output is normal, the risk of thromboembolism is low.

During thromboembolism attacks, cardiac imaging methods of heart and organ where the embolic incident occurred establishes the diagnosis and determines the relation of the heart and the situation. Built-in fibrin clusters which can be viewed by at the atrial surfaces of the mitral prosthesis and at ventricular surfaces of the aortic prosthesis often cause thromboembolic events. These clusters can be monitored easily by TEE. In a study of Isada et al. [10], fibrin clusters are observed that $18 \%$ of the mitral prostheses, and they has been caused hemodynamic problems in 53\% of it (Figure 3).

In order to establish appropriate treatment, if it is clinically safe, target anticoagulation level should be increased or an antiplatelet drug should be added. In order to prevent further thromboembolic events, anticoagulant therapy should be optimized as described above, patient should be followed with neurology, and predisposing factors should be treated or reversed [1].

\section{PROSTHETIC VALVE REGURGITATION}

There is a small amount of physiological valvular regurgitation in all prosthetic valves. The markers of physiological valvular regurgitation are as follows: 1: Jet area $<2 \mathrm{~cm}^{2}$ and jet length $<2.5 \mathrm{~cm}$ in mitral position; 2: Jet area $<1 \mathrm{~cm}^{2}$ and the jet length is $<1.5 \mathrm{~cm}$ in aortic position; 3: Blood flow properties is intended for the characteristic of the prosthesis: (Single-center jet, in Medtronic Hall, two different jets with different sizes in Björk-Shiley, 2 side and 1 central jet in St. Jude Medical's, etc.). Markers of the pathologic regurgitation are valvular regurgitant jets which does not cover the features above and all the jets outside from the valve ring (paravalvuler regurgitant jets) [4]. All non-physiological and paravalvular leaks are cause of re-surgical intervention.

Paravalvular regurgitant jets usually depends on the separation of the PHV and annulus (valve dehiscence) (Figures 4(A) and (B) and Figures 5(A) and (B)) caused by infective endocarditis. However, it may caused by improper valve placement and suture line breakage [4]. In severe paravalvular mitral regurgitation, Coanda effect in TEE (circular jet motion on the walls of the left atrium) can be observed [8]. Paravalvular jets are important because they require replacement of PHV or repair of 


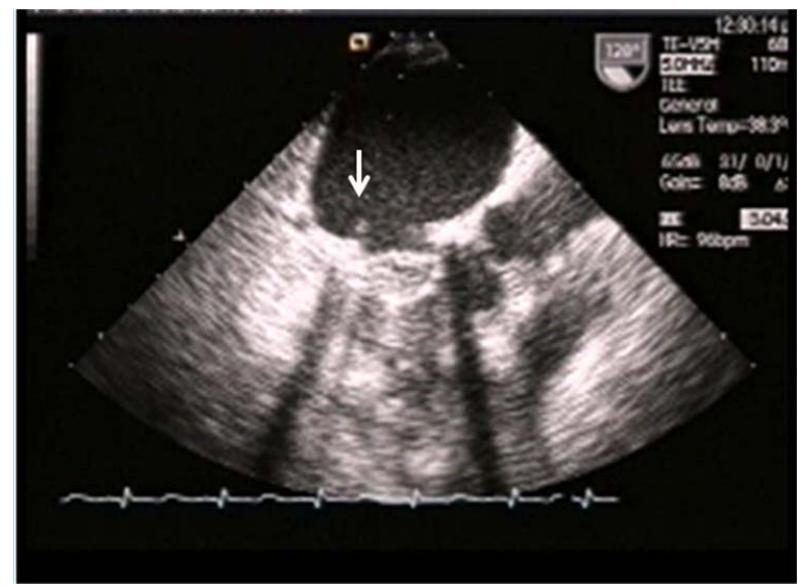

Figure 3. Arrow points fibrin cluster at mechanical mitral valve prosthesis.
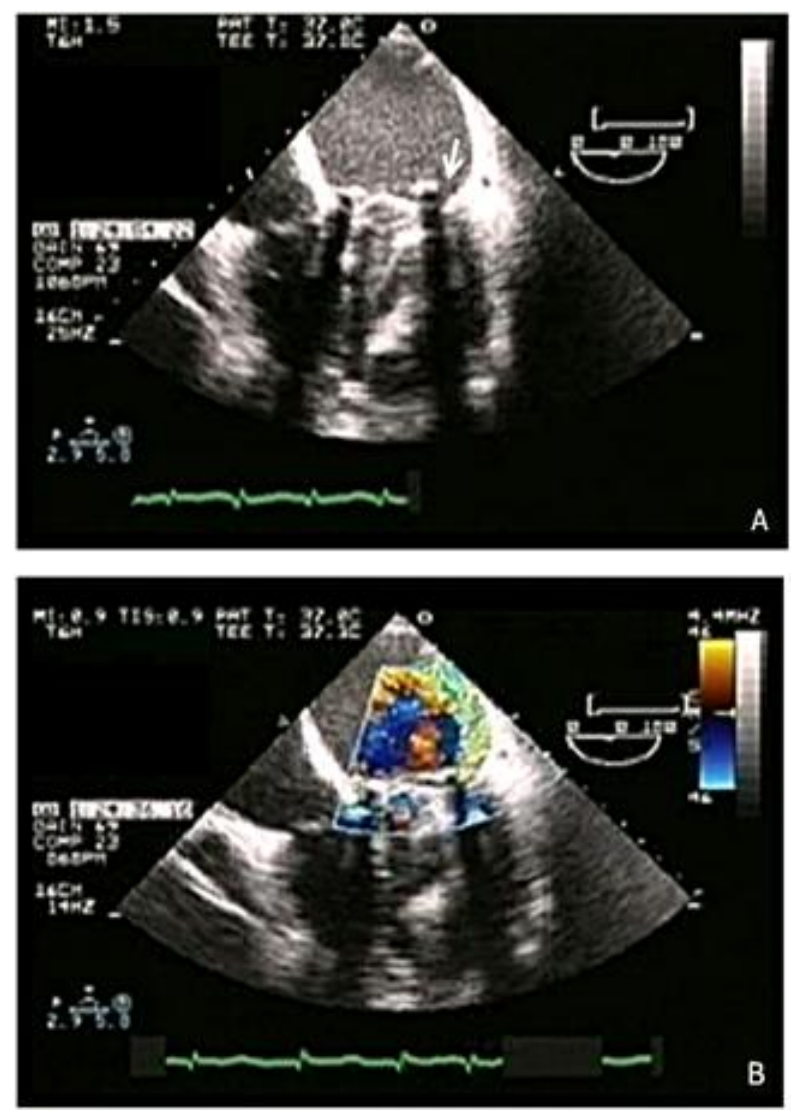

Figure 4. (A) Dehiscence of a mitral mechanical prosthetic valve (arrow); (B) Severe paravalvular mitral regurgitation and Coanda effect.

defect by surgical methods. Normally, most of the PHV may accompany a mild chronic hemolytic anemia. Paravalvular regurgitant jets, however, often lead to refractory hemolysis and hemolytic anemia. Severe hemolytic anemia, should indicate paravalvular regurgitation.

For the diagnosis of prosthetic aortic valve regurgitation, a comprehensive assessment of two-dimensional/
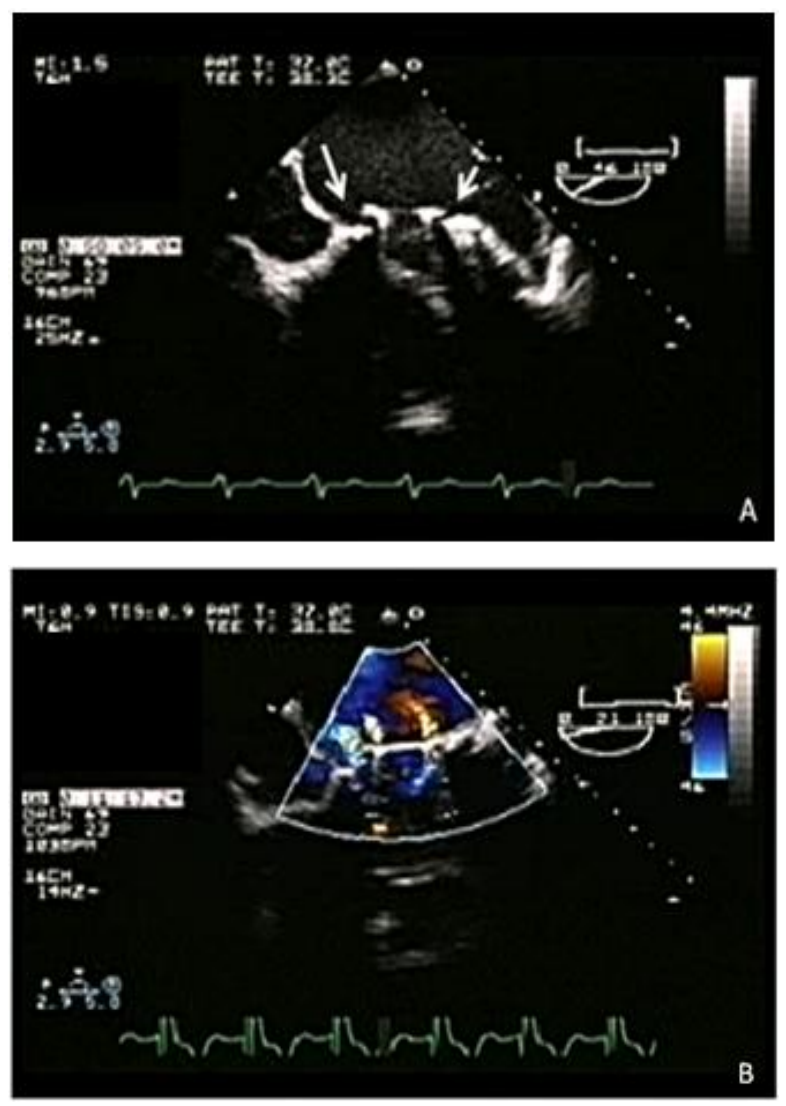

Figure 5. (A) Valve dehiscence in a prosthetic mitral valve (arrow); (B) Double tilting disc mechanical mitral prosthesis with 3 regurgitation jets. The first jet at the left is a paravalvular jet, and results from the valve dehiscence. The other two jets are physiological.

color Doppler echocardiography by TTE and TEE, should be done. Grading is the same as native valves. Ventricular size and function are important determinants of severity. Measurements of short axis jet length and jet area are shown to be compatible with angiographic severity of aortic regurgitation $(r=0.93$ and 0.91$)$ [8]. Eccentric jets may cause overestimation of the severity of regurgitation caused by the prosthesis. TEE, can provide insight into primary mechanical prosthetic valve regurgitation, informs about the structure, (broken leaflet, valve ring separation, vegetations, abscesses, fistulas next to the valve) in detail. Generally, by a careful TTE and/or TEE, separation point of the valve and pathological blood jet can be demonstrated easily. On the other hand, (left ventricle outflow tract area/jet area) is easy to measure due to very good visibility of the left ventricular outflow tract by TEE. In addition, evaluation of the ascending aorta aneurysms and dissection should be a routine part of the TEE [8]. Indicators of severe aortic regurgitation are: 1 : jet pressure half-time $\leq 250 \mathrm{~ms}$; 2 : restictive mitral filling pattern in acute aortic regurgitation; 3 : diastolic flow reversal in the descending aorta; 4: regur- 
gitant fraction $\geq 55 \%$ [4].

Prosthetic mitral valve regurgitation, should also be evaluated by multiple methods. When assessing hemodynamic effects of mitral regurgitation, whether the regurgitation is acute or not is very important. For example, even a moderate degree of acute mitral prosthetic regurgitation may lead to pulmonary edema, because of the inflexible left atrium. In contrast, patients with chronic and severe mitral prosthetic regurgitation may have no symptoms due to compensatory mechanisms. Evaluation of mitral regurgitation of a mechanical prosthesis by TTE is limited due to reverberations. This problem is less pronounced in bioprosthesis. So, as a general rule, if pathological mitral regurgitation is suspected, regardless of the type of prosthesis, TEE should be done. Markers of severe prosthetic mitral regurgitation: 1: Increased mitral inflow velocity $(\geq 2.5 \mathrm{~m} / \mathrm{s})$ and normal mitral valve pressure half time $(\leq 150 \mathrm{~ms})$; 2: Intense Continuous wave Doppler mitral regurgitation jet; 3: Regurgitant fraction $\geq 55 \%$; 4 : effective regurgitant orifice area $\geq 0.34$ $\mathrm{cm}^{2}, 5$ : systolic pulmonary venous reversals. Proximal isovelocity surface area (PISA), which can be determined not only by TTE, but by TEE, highlights a severe mitral regurgitation. In a study of prosthetic mitral regurgitation, PISA was compared with the Doppler signal intensity and the intensity of the jet in the left atrium and PISA was found more sensitive but less specific [11]. Unexplained worsening in clinical status or new pulmonary hypertension, may be signs of severe mitral regurgitation [8]. Pulmonary venous Doppler flow is suggestive of the diagnosis of severe prosthetic mitral valve regurgitation, disappearance of systolic wave and return of systolic flow reversals in favor of seriousness. Pulmonary venous flow reversal, more evident in acute prosthetic mitral regurgitation, compared to the chronic prosthetic mitral regurgitation. However, there are no standards available for pulmonary venous flows for the evaluation of prosthetic mitral regurgitation. Coanda effect in TEE [8] indicates severe mitral regurgitation in valve dehiscence (Figure 4(B)).

\section{REFERENCES}

[1] Nishimura, R.A., Carabello, B.A., Faxon, D.P., Freed, M.D., Lytle, B.W., O'Gara, P.T., et al. (2008) ACC/AHA 2008 Guideline update on valvular heart disease: Focused update on infective endocarditis: A report of the American College of Cardiology/American Heart Association Task Force on Practice Guidelines endorsed by the Society of Cardiovascular Anesthesiologists, Society for Cardiovascular Angiography and Interventions, and Society of Thoracic Surgeons. Journal of the American College of Cardiology, 52, 676-685. doi:10.1016/j.jacc.2008.05.008

[2] Girard, S.E., Miller Jr., F.A., Orszulak, T.A., Mullany, C.J., Montgomery, S., Edwards, W.D., et al. (2001) Reoperation for prosthetic aortic valve obstruction in the era of echocardiography: Trends in diagnostic testing and comparison with surgical findings. Journal of the American College of Cardiology, 37, 579-584. doi:10.1016/S0735-1097(00)01113-X

[3] Cannegieter, S.C., Torn, M. and Rosendaal F.R. (1999) Oral anticoagulant treatment in patients with mechanical heart valves: How to reduce the risk of thromboembolic and bleeding complications. Journal of Internal Medicine, 245, 369-374.

[4] Oh, J.K., Seward, J.B. and Tajik, A.J. (2007) Prosthetic valve evaluation. In: Oh, J.K., Seward, J.B. and Tajik, A.J., Eds., The Echo Manual, 3rd Edition, Lippincott, Williams \& Wilkins, Philadelphia, 226-242.

[5] Barbetseas, J., Nagueh, S.F., Pitsavos, C., Toutouzas, P.K., Quinones, M.A. and Zoghbi, W.A. (1998) Differentiating thrombus from pannus formation in obstructed mechanical prosthetic valves: An evaluation of clinical, transthoracic and transesophageal echocardiographic parameters. Journal of the American College of Cardiology, 32, 1410-1417. doi:10.1016/S0735-1097(98)00385-4

[6] Roudaut, R., Lafitte, S., Roudaut, M.F., Courtault, C., Perron, J.M., Jais, C. et al. (2003) Fibrinolysis of mechanical prosthetic valve thrombosis: A single-center study of 127 cases. Journal of the American College of Cardiology, 19, 653-658.

[7] Rizzoli, G., Guglielmi, C., Toscano, G., Pistorio, V., Vendramin, I., Bottio, T., et al. (1999) Reoperations for acute prosthetic thrombosis and pannus: An assessment of rates, relationship and risk. Eur J Cardiothorac Surg., 16, 74-80. doi:10.1016/S1010-7940(99)00124-4

[8] Zabalgoitia, M. (2007) Echocardiographic recognition and quantitation of prosthetic valve disease. In: Otto, C., Ed., The Practice of Clinical Echocardiography, 3rd Edition, Saunders Elsevier, Philadelphia, 577-604. doi:10.1016/B978-1-4160-3640-1.50029-0

[9] Butchart, E.G., Moreno, de la Santa, P., Rooney, S.J. and Lewis, P.A. (1995) Arterial risk factors and ischemic cerebrovascular events after aortic valve replacement. Journal of Heart Valve Disease, 4, 1-8.

[10] Isada, L.R., Torelli, J.N., Stewart, W.J. and Klein A.L. (1994) Detection of fibrous strands on prosthetic mitral valves with transesophageal echocardiography: Another potential embolic source. Journal of the American College of Cardiology, 7, 641-645. doi:10.1016/0735-1097(95)00375-4

[11] Nellessen, U., Masuyama, T., Appleton, C.P., Tye, T. and Popp, R.L. (1989) Mitral prosthesis malfunction. Comparative Doppler echocardiographic studies of mitral prostheses before and after replacement. Circulation, 79, 330336. doi:10.1161/01.CIR.79.2.330 